\title{
Multiple Disulfide-Bonded States of Native Proteins: Estimate of Number Using Probabilities of Disulfide Bond Formation
}

\author{
Philip J. Hogg 1,2 (1) \\ 1 The Centenary Institute, Camperdown, NSW 2050, Australia; phil.hogg@sydney.edu.au; \\ Tel.: +61-2-8627-4716 \\ 2 NHMRC Clinical Trials Centre, University of Sydney, Camperdown, NSW 2006, Australia \\ Academic Editor: Michio Iwaoka \\ Received: 10 November 2020; Accepted: 2 December 2020; Published: 4 December 2020
}

\begin{abstract}
The polypeptide backbone of proteins is held together by two main types of covalent bonds: the peptide bonds that link the amino acid residues and the disulfide bonds that link pairs of cysteine amino acids. Disulfide bonds form as a protein folds in the cell and formation was assumed to be complete when the mature protein emerges. This is not the case for some secreted human blood proteins. The blood clotting protein, fibrinogen, and the protease inhibitor, $\alpha 2$-macroglobulin, exist in multiple disulfide-bonded or covalent states in the circulation. Thousands of different states are predicted assuming no dependencies on disulfide bond formation. In this study, probabilities for disulfide bond formation are employed to estimate numbers of covalent states of a model polypeptide with reference to $\alpha 2$-macroglobulin. When disulfide formation is interdependent in a protein, the number of covalent states is greatly reduced. Theoretical estimates of the number of states will aid the conceptual and experimental challenges of investigating multiple disulfide-bonded states of a protein.
\end{abstract}

Keywords: disulfide bond; cystine; cysteine; probability; allosteric

\section{Introduction}

Most disulfide bonds in mammalian proteins were acquired in vertebrate ancestors and retained as the proteins evolved [1]. Mammalian membrane and secreted proteins are particularly rich in disulfide bonds, although they are being found in proteins in all subcellular compartments. Disulfides form during maturation of proteins in the cell and bond formation has been assumed to be complete in the mature functional protein. Our recent findings indicate that this is not the case for some, perhaps many, secreted proteins [2].

Over 180,000 disulfide bonds in three-dimensional protein structures have been defined. Protein crystallization is a very exacting type of protein purification and favors the most stable, lowest energy forms of a protein. This will typically be proteins where all disulfide bonds are intact. In addition, isolating a protein that contains closely spaced cysteine thiols and preparing it for structural studies in ambient oxygen can result in oxidation of the thiols to a disulfide bond. It is not unexpected, therefore, that disulfide bonds are almost invariably intact in experimentally determined protein structures. Largely based on this information, it has been assumed that disulfide bonds are inert structural motifs that help proteins fold correctly and stabilise the tertiary and quaternary structure. I reported that two circulating blood proteins exist and function as multiple disulfide-bonded forms [2].

The redox state of the disulfide bonds in the blood clotting protein, fibrinogen, and the protease inhibitor, $\alpha 2$-macroglobulin, was determined using differential cysteine alkylation and mass spectrometry. The proteins were analysed in their ex vivo plasma environment and the findings 
represent the states of the circulating proteins. Thirteen fibrinogen disulfide bonds and twelve $\alpha 2$-macroglobulin disulfides are $10-50 \%$ and $10-70 \%$ reduced in the blood of healthy human donors, respectively. The functional relevance of the disulfide lability for fibrinogen conversion to fibrin polymer was explored. We observed that disulfides form upon fibrin polymerization and this is important for a functional fibrin matrix. Moreover, the covalent states of fibrinogen in plasma are influenced by physiologically relevant fluid shear forces, indicating that the different states can change in response to an external stimulus.

In this work, I have explored the question of how many disulfide-bonded forms of proteins exist. The redox state of 13 of the 17 bonds in fibrinogen and all 12 disulfides in $\alpha 2$-macroglobulin have been determined [2]. As we do not have a complete picture of the disulfide bonds in fibrinogen, I have focused on the results for $\alpha 2$-macroglobulin. Using probabilities for bond formation, the numbers of $\alpha 2$-macroglobulin covalent states is estimated and discussed.

\section{Results and Discussion}

$\alpha 2-$ Macroglobulin is an abundant broad-spectrum endopeptidase inhibitor [3] produced by liver hepatocytes and macrophages that circulates in blood and functions in several biological systems [4]. Human $\alpha 2$-macroglobulin is a $720 \mathrm{kDa}$ homo-tetrameric glycoprotein that targets proteases of the four major classes via a 'venus flytrap' mechanism [5]. Proteases cleave peptide bonds in the 'bait' region and become entrapped due to large conformational transitions in the inhibitor. These transitions expose a cysteine-glutamine thioester bond in $\alpha 2$-macroglobulin that is cleaved by lysine residues of proteases, resulting in their covalent attachment to the inhibitor. Proteases form 1:1 or 2:1 complex with the tetramer and remain accessible for small substrates or inhibitors. The protease-induced conformational transitions in $\alpha 2$-macroglobulin also expose the receptor binding domain that interacts with specific receptors on cells, leading to endocytosis of the complex and degradation in lysosomes.

The $\alpha 2$-macroglobulin monomer contains 11 intra-chain disulfide bonds and two inter-chain bonds involving Cys278 and Cys241 that link dimers. Seven disulfides (C48-C86, C251-C299, C269-C287, C470-C563, C595-C771, C821-C849 and C847-C883) are in the seven macroglobulin-like domains, which are antiparallel $\beta$-sandwiches comprising a three- and a four-stranded sheet. The C642-C689 disulfide is adjacent to the bait region (residues 690-728), while the C1079-C1127 bond is in the thiol-ester domain that contains the C927-Q975 thioester bond. The C921-C1321 disulfide is in the CUB domain and the receptor binding domain contains the C1352-C1467 bond.

Disulfide bonds can adopt 20 different conformations defined by the geometry of the five dihedral angles that describe the cystine residue [6]. Two of the 20 disulfide conformations, the -RHstaple and $-/+$ RHhook bonds, are naturally strained due to stretching of the S-S bond and neighbouring $\alpha$ angles [6-8]. It is noteworthy that 5 of the $12 \alpha 2$-macroglobulin disulfides have a stressed -RHstaple conformation (Table 1), suggesting that the disulfide landscape of the protein is strained. This may contribute to the general disulfide lability in the protein.

The $12 \alpha 2$-macroglobulin disulfide bonds ranged from a mean of 33 to $92 \%$ formed or oxidized in eight healthy human donors ( 3 male, 5 female, 18-48 years old) (Figure 1) [2]. There was very little donor-to-donor variation in the redox states of the disulfides. Coefficients of variation ranged from $2.5 \%$ for the $\mathrm{C} 642-\mathrm{C} 689$ disulfide to $10.8 \%$ for the C847-C883 bond. A protein containing $n$ disulfide bonds, where the bonds are either formed or broken, has $2^{n}$ possible disulfide-bonded states. In the case of the $12 \alpha 2$-macroglobulin disulfides, this equates to maximum 4096 possible disulfide-bonded states of the protein. This estimate assumes that formation of any disulfide bond in the protein is independent of any other, which is likely not the case in a protein the size and complexity of $\alpha 2$-macroglobulin. Using probabilities for bond formation, I estimate the theoretical numbers of $\alpha 2$-macroglobulin covalent states. 
Table 1. Conformation of the $\alpha 2$-macroglobulin disulfide bonds and fraction of the bonds that are oxidized in protein populations from healthy human donors. The disulfide conformations were determined using the Disulfide Bond Analysis tool [9] and PDB identifier 4acq structure of $\alpha 2$-macroglobulin homo-tetramer [5]. Two of the disulfides have different conformations in 1 of the 4 molecules of the tetramer. The fraction oxidized are the mean values of eight donors (data from Figure 1a) and are equivalent to the probability that the disulfide bond is formed in the population of 22-macroglobulin molecules.

\begin{tabular}{|c|c|c|}
\hline Q2-Macroglobulin Disulfide & Conformation & Fraction Oxidized \\
\hline C48-C86 & -RHstaple & 0.92 \\
\hline C251-C299 & -LHhook & 0.85 \\
\hline C269-C287 & -RHstaple & 0.87 \\
\hline C278-C431 & -RHstaple & 0.89 \\
\hline C470-C563 & -RHspiral & 0.69 \\
\hline C595-C771 & -RHstaple & 0.75 \\
\hline C642-C689 & -LHstaple & 0.33 \\
\hline C821-C849 & -LHstaple & 0.84 \\
\hline C847-C883 & +/-LHhook & 0.82 \\
\hline C921-C1321 & -RHstaple & 0.85 \\
\hline C1079-C1127 & -RHspiral & 0.81 \\
\hline C1352-C1467 & +/-LHstaple & 0.76 \\
\hline
\end{tabular}

The C278-C431 inter-chain disulfide linking the $\mathrm{C}$ and $\mathrm{D}$ chains of the homo-tetramer has a -LHspiral conformation. The C642-C689 intra-chain disulfide in the D chain of the homo-tetramer has a +/-LHstaple conformation.

(a)

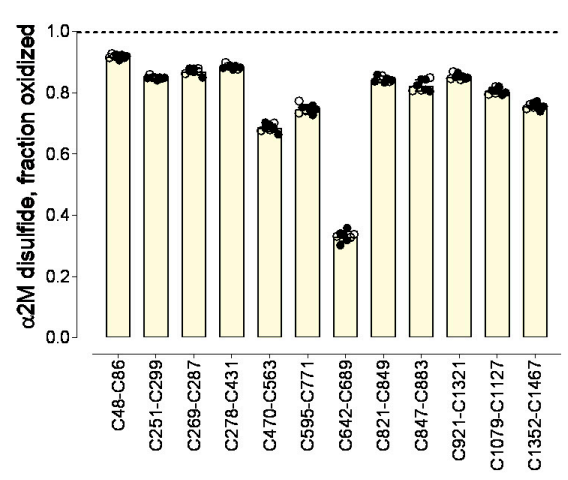

(b)

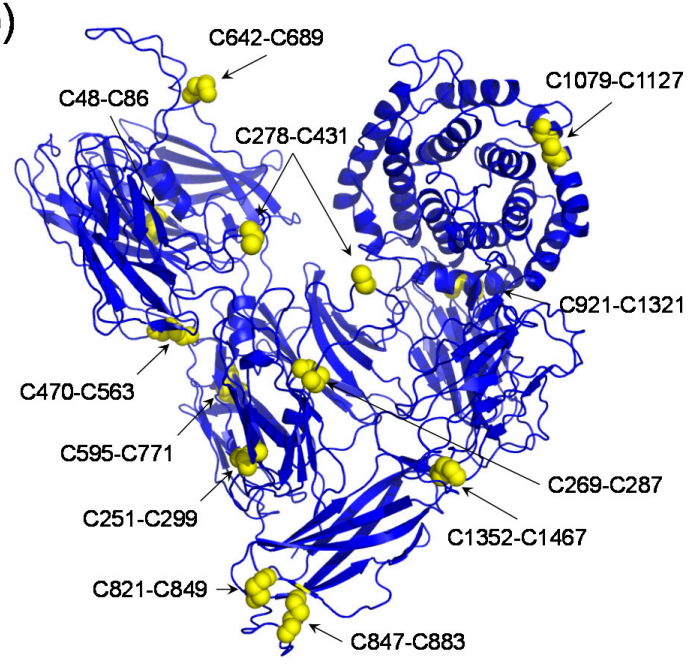

Figure 1. $\alpha 2$-Macroglobulin exists in multiple disulfide-bonded states in the circulation. (a) Redox states of the $12 \alpha 2$-macroglobulin disulfide bonds in eight healthy human donors (5 female-solid symbols; 3 male-open symbols). The bars and errors are mean \pm SD. (b) Ribbon structure of $\alpha 2$-macroglobulin monomer and the positions of the 12 disulfide bonds [5] (PDB identifier 4acq). Disulfide bond cysteines are shown as yellow spheres. The $\mathrm{C} 278$ and $\mathrm{C} 431$ residues form the inter-dimer disulfides. Figure adapted from Figure 6 of Butera and Hogg [2].

The mean oxidized disulfide bond fraction for the individual $\alpha 2$-macroglobulin disulfides represent the fraction of the bond that is formed in the population of molecules (Figure 1a). Probability $(P)$ is defined as the extent to which an event is likely to occur, measured by the ratio of the favorable cases to the whole number of cases possible. The event for disulfides is binary, that is the bond is either 
formed (oxidized) or unformed (reduced) and the favorable case for this analysis is the formed bond. Approximately $5 \mu \mathrm{g}$ of plasma $\alpha 2$-macroglobulin was analysed for each donor [2] that equates to $\sim 10^{12}$ molecules of the protein, which is an exhaustive sampling of possible cases. Therefore, the mean oxidized fraction for the individual $\alpha 2$-macroglobulin disulfides represents the probability that the bond is formed in the population of molecules (Table 1). To explore the variables involved in estimating types and numbers of $\alpha 2$-macroglobulin states, a model polypeptide containing five disulfide bonds is simulated.

A polypeptide containing five disulfide bonds, where the bonds are either formed or broken, can exist in $32\left(2^{5}\right)$ possible disulfide-bonded states (Figure 2). These different states are represented in cartoon form in Figure $2 b$. It is assumed that the redox potential of the system is unchanging, so the redox states of the bonds will be static. This is a reasonable assumption for proteins that function in plasma-an environment with a stable redox potential due to small molecule and protein thiol buffering mechanisms. Listed in Table 2 are all combinations of states containing 1, 2, 3, 4 or 5 disulfide bonds, the disulfide isomeric states that contain the bond or bonds using the numbering in Figure $2 b$, and the probability that the states exists in the population assuming no special dependence between states. The probability is the ratio of the number of states to total number of states (32). For example, there are 10 possible states where only 2 of the 5 bonds are formed and each state occurs in 8 of the 32 possible isomers, so the probability of an isomer containing only 2 disulfide bonds is $8 / 32$ or 0.25 . For the 5 situations, that is formation of 1 bond given formation of 1, 2, 3 or 4 others, the following conditional probabilities apply:

$$
\begin{gathered}
\mathrm{P}(1 \text { bond })=0.5 \\
\mathrm{P}(1 \text { bond } \mid 1 \text { other })=0.25 \\
\mathrm{P}(1 \text { bond } \mid 1 \text { other })=0.25 \\
\mathrm{P}(1 \text { bond } \mid 2 \text { others })=0.125 \\
\mathrm{P}(1 \text { bond } \mid 3 \text { others })=0.0625 \\
\mathrm{P}(1 \text { bond } \mid 4 \text { others })=0.03125
\end{gathered}
$$

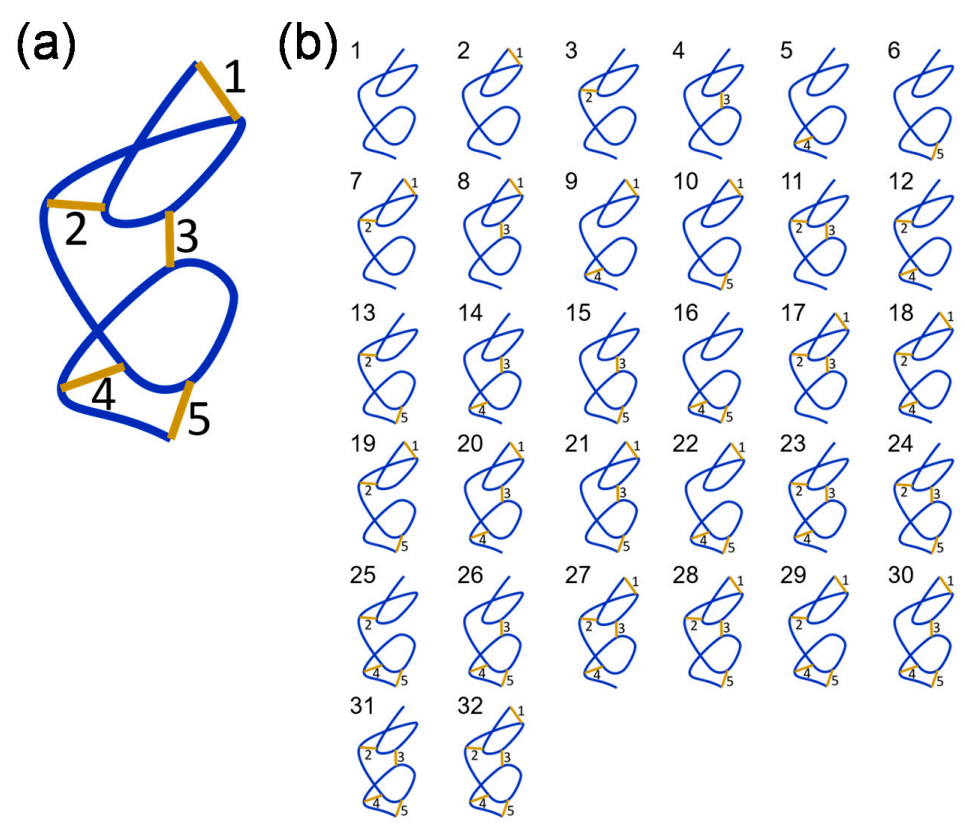

Figure 2. A protein containing $n$ disulfide bonds has $2^{n}$ possible disulfide-bonded states. (a) A cartoon polypeptide containing 5 disulfide bonds. (b) A polypeptide containing 5 disulfide bonds, where the bonds are either formed or broken, can exist in 32 possible disulfide-bonded states. Figure adapted from Supplementary Figure S3 of Butera and Hogg [2]. 
Table 2. A polypeptide containing 5 disulfide bonds, where the bonds are either formed or broken, can exist in 32 possible disulfide states. Shown are all combinations of states containing only 1, 2, 3, 4 or 5 disulfide bonds, the disulfide isomers that contain the bond or bonds using the numbering in Figure $2 b$, and the probability that the disulfide bond or bonds exist in the population assuming no dependencies between states.

\begin{tabular}{|c|c|c|}
\hline Disulfides Formed & Disulfide Isomers Containing the Bond(s) & Probability \\
\hline 1 & $2,7,8,9,10,17,18,19,20,21,22,27,28,29,30,32$ & 0.5 \\
\hline 2 & $3,7,11,12,13,17,18,19,23,24,25,27,28,29,31,32$ & 0.5 \\
\hline 3 & $4,8,11,14,15,17,20,21,23,24,26,27,28,30,31,32$ & 0.5 \\
\hline 4 & $5,9,12,14,16,18,20,22,23,25,26,27,29,30,31,32$ & 0.5 \\
\hline 5 & $6,10,13,15,16,19,21,22,24,25,26,28,29,30,31,32$ & 0.5 \\
\hline 1,2 & $7,17,18,19,27,28,29,32$ & 0.25 \\
\hline 1,3 & $8,17,20,21,27,28,30,32$ & 0.25 \\
\hline 1,4 & $9,18,20,22,27,29,30,32$ & 0.25 \\
\hline 1,5 & $10,19,21,22,28,29,30,32$ & 0.25 \\
\hline 2,3 & $11,17,23,24,27,28,31,32$ & 0.25 \\
\hline 2,4 & $12,18,23,25,27,29,31,32$ & 0.25 \\
\hline 2,5 & $13,19,24,25,28,29,31,32$ & 0.25 \\
\hline 3,4 & $14,20,23,26,27,30,31,32$ & 0.25 \\
\hline 3,5 & $15,21,24,26,28,30,31,32$ & 0.25 \\
\hline 4,5 & $16,22,25,26,29,30,31,32$ & 0.25 \\
\hline $1,2,3$ & $17,27,28,32$ & 0.125 \\
\hline $1,2,4$ & $18,27,29,32$ & 0.125 \\
\hline $1,2,5$ & $19,28,29,32$ & 0.125 \\
\hline $1,3,4$ & $20,27,30,32$ & 0.125 \\
\hline $1,3,5$ & $21,28,30,32$ & 0.125 \\
\hline $1,4,5$ & $22,29,30,32$ & 0.125 \\
\hline $2,3,4$ & $23,27,31,32$ & 0.125 \\
\hline $2,3,5$ & $24,28,31,32$ & 0.125 \\
\hline $2,4,5$ & $25,29,31,32$ & 0.125 \\
\hline $3,4,5$ & $26,30,31,32$ & 0.125 \\
\hline $1,2,3,4$ & 27,32 & 0.0625 \\
\hline $1,2,3,5$ & 28,32 & 0.0625 \\
\hline $1,2,4,5$ & 29,32 & 0.0625 \\
\hline $1,3,4,5$ & 30,32 & 0.0625 \\
\hline $2,3,4,5$ & 31,32 & 0.0625 \\
\hline $1,2,3,4,5$ & 32 & 0.03125 \\
\hline
\end{tabular}

For the general case for any number of disulfides, the probability that a given number (n) of disulfide bond(s) will be formed in a protein, assuming there is no special dependence in formation of bonds, is:

$$
P(n)=2^{-n}
$$

For example, if a protein contains 20 disulfide bonds, the probability that all bonds are formed, assuming no special dependencies, is very small at $9.5 \times 10^{-7}$. This analysis assumes no dependencies 
on formation of the bonds. This is an unlikely scenario in complex proteins containing many disulfide bonds, such as $\alpha 2$-macroglobulin and fibrinogen. For instance, one or more bonds might be required to form before another bond can form. These situations are illustrated using the model polypeptide and two different special conditions.

The first condition assumes that disulfide number 5 forms only if disulfide number 2 has formed. This results in a reduction in the total number of disulfide states from 32 to 24 (Figure 3(aii)). The second condition assumes that disulfide number 2 must form before any other bonds can form and disulfide number 5 forms only if disulfide number 4 has formed. In this situation, the number of disulfide states reduces to a total of 13 (Figure 3(aiii)). The probability that each of the five disulfide bonds are formed in the different scenarios is shown in Figure 3b. It is apparent that special conditions change the probabilities for disulfide formation from a value of 0.5 for all bonds if there are no dependencies (Figure 3(ai)) to values as low as $\sim 0.3$ and high as $\sim 0.9$ if there are particular dependencies for bond formation (Figure 3(aiii)).

(a)

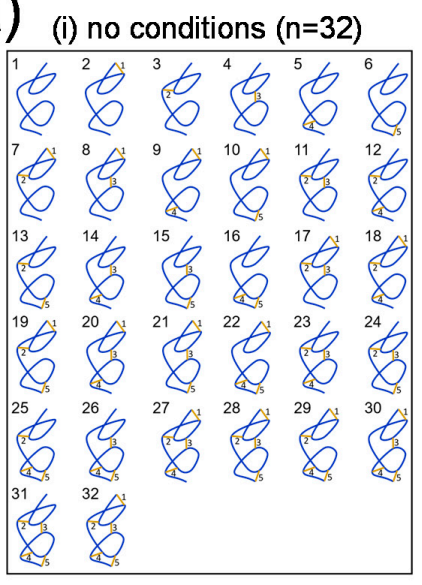

(ii) 5 only if $2(n=24)$

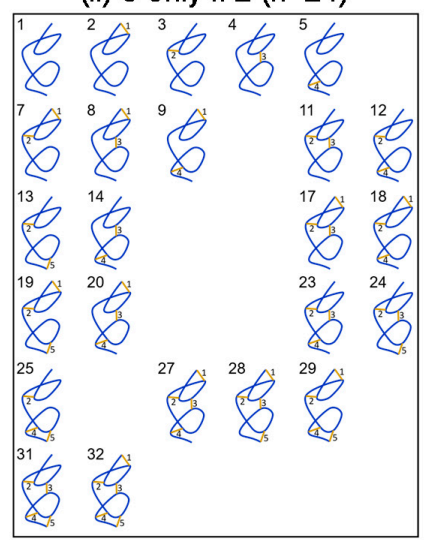

(iii) must 2 \& 5 only if $4(n=13)$

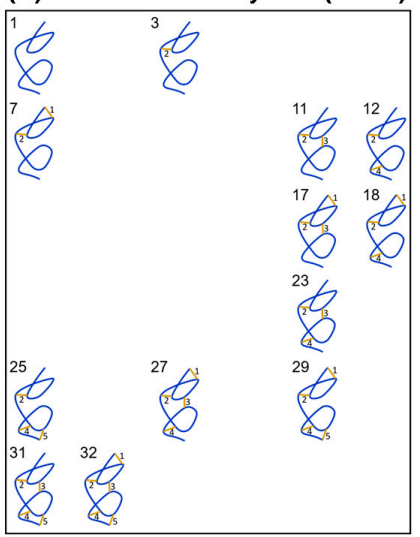

(b)

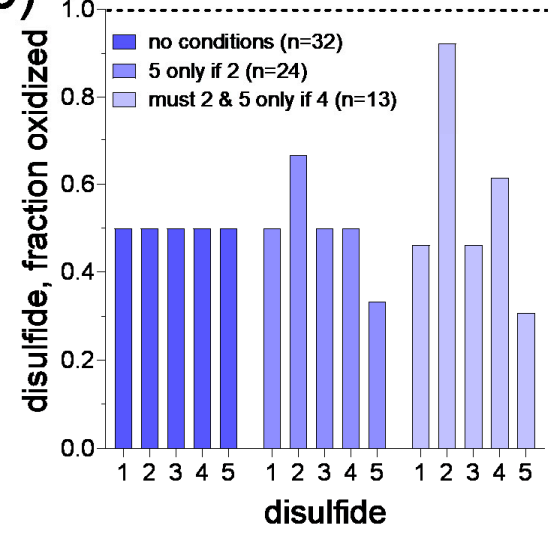

Figure 3. Special conditional formation of disulfide bonds. (a) A polypeptide containing 5 disulfide bonds, where the bonds are either formed or broken, can exist in 32 possible disulfide states (part (i)). If the condition is applied that disulfide number 5 only forms if disulfide number 2 has formed, the total number of disulfide states reduces from 32 to 24 (part (ii)). If the condition is applied that disulfide number 2 must form before any other bonds can form and disulfide number 5 forms only if disulfide number 4 has formed, the total number of disulfide states reduces further to 13 (part (iii)). (b) Fraction of the 5 disulfide bonds that are oxidized in the scenarios in part A, which represents the probability that the disulfide bonds are formed in the population. This representation mirrors the expression of the experimental data shown in Figure 1a. 
Note that the probability of a state containing any number of bonds is the product of the probabilities for each of the bonds:

$$
\mathrm{P}(\mathrm{a} \& \mathrm{~b} \& \mathrm{c} \ldots)=\mathrm{P}(\mathrm{a}) \times \mathrm{P}(\mathrm{b}) \times \mathrm{P}(\mathrm{c}) \times \ldots
$$

This is illustrated in Table 3, which lists the probabilities for formation of only 2 or 3 bonds when there are no conditions on bond formation and for the two special cases. These calculations are applied to the 12 experimentally determined bond states in $\alpha 2$-macroglobulin.

Table 3. Probabilities for formation of only 2 or 3 bonds in a polypeptide containing 5 disulfide bonds when there are no conditions on bond formation, and for two special cases. The disulfide isomers that contain the 2 or 3 bonds uses the numbering in Figure 2b.

\begin{tabular}{|c|c|c|c|c|}
\hline Disulfide & Probability & Disulfides Formed & Disulfide Isomers & Probability * \\
\hline \multicolumn{5}{|c|}{ No conditions } \\
\hline 1 & 0.50 & 1,3 & $8,17,20,21,27,28,30,32$ & 0.25 \\
\hline 2 & 0.50 & $1,3,5$ & $21,28,30,32$ & 0.125 \\
\hline 3 & 0.50 & & & \\
\hline 4 & 0.50 & & & \\
\hline 5 & 0.50 & & & \\
\hline \multicolumn{5}{|c|}{ Bond number 5 only forms if bond number 2 has formed } \\
\hline 1 & 0.50 & 1,3 & $8,17,20,27,28,32$ & 0.25 \\
\hline 2 & 0.67 & $1,3,5$ & 28,32 & 0.083 \\
\hline 3 & 0.50 & & & \\
\hline 4 & 0.50 & & & \\
\hline 5 & 0.33 & & & \\
\hline \multicolumn{5}{|c|}{ Bond number 2 must form and bond number 5 forms only if bond number 4 is formed } \\
\hline 1 & 0.46 & 1,3 & $17,27,32$ & 0.213 \\
\hline 2 & 0.92 & $1,3,5$ & 32 & 0.066 \\
\hline 3 & 0.46 & & & \\
\hline 4 & 0.62 & & & \\
\hline 5 & 0.31 & & & \\
\hline
\end{tabular}

From Equation (3), the probability that all 12 bonds are formed in $\alpha 2$-macroglobulin, assuming there is no special dependence in formation of bonds, is the product of the probabilities for each bond. For the 12 values of mean oxidized state (Table 1 ), this equates to a probability of 0.037 . In other words, just $3.7 \%$ of circulating plasma $\alpha 2$-macroglobulin is predicted to be fully oxidized. If disulfide bond formation in $\alpha 2$-macroglobulin has special conditions, however, the total number of disulfide isomeric states will be reduced, and the incidence of fully oxidized protein will increase. On the other hand, this analysis assumes that a fully oxidized protein can indeed exist, which has not been demonstrated experimentally. It is possible, for instance, that one bond will not form if one or more other bonds have formed and so a fully oxidized protein cannot exist.

For the $12 \alpha 2$-macroglobulin disulfides, 8 have probabilities of formation $>0.8$ while 4 of the 12 have probabilities of formation from 0.33 to 0.76 (Figure 1a, Table 1). This indicates that the majority of the $\alpha 2$-macroglobulin disulfides are favored to form. The total number of $\alpha 2$-macroglobulin states, therefore, is much smaller than the 4096 predicted assuming no dependencies on disulfide bond formation. Even so, a hundred-fold fewer states for instance would mean that the protein circulates in 
$\sim 40$ different covalent forms, which is a potential complexity of protein function not envisaged for a single fully oxidized state. An example of this complexity could be the fate of the C642-C689 disulfide.

The C642-C689 bond adjacent to the residue 690-728 bait region is the most reduced bond in the protein, with only 1 in 3 molecules containing an oxidized bond (Table 1). This suggests that the C642-C689 disulfide might be an allosteric bond, which is a different type of disulfide bond to that described herein. Allosteric disulfide bonds are cleaved in the mature protein and control function through conformation changes as a result of bond cleavage $[6,7,10]$. These bonds are found on or near the protein's surface so they are accessible to factors, such as the oxidoreductases, that manipulate their redox state [11]. The C689 residue of the C642-C689 bond is the only one of the 24 disulfide cysteines that is freely accessible to solvent in the crystal structure of the $\alpha 2$-macroglobulin homo-tetramer (Figure 4). It will be of interest to determine if cleavage of the C642-C689 bond by a vascular thiol isomerase [11] controls protease cleavage of the bait region. The other 11 disulfide bonds in mature $\alpha 2$-macroglobulin are predicted to be inaccessible to oxidoreductases, which implies their redox state is set during folding and maturation, as is the case for most of the disulfide bonds in fibrinogen [2]. It is also possible that redox state of the C642-C689 bond is controlled by formation of one or more other bonds. For instance, the probability that both the C642-C689 and nearby C48-C 86 bonds are both formed in the population of molecules is $0.30(0.33 \times 0.92)$. It may be, for example, that both disulfides are required to be reduced or oxidised to inhibit certain proteases.

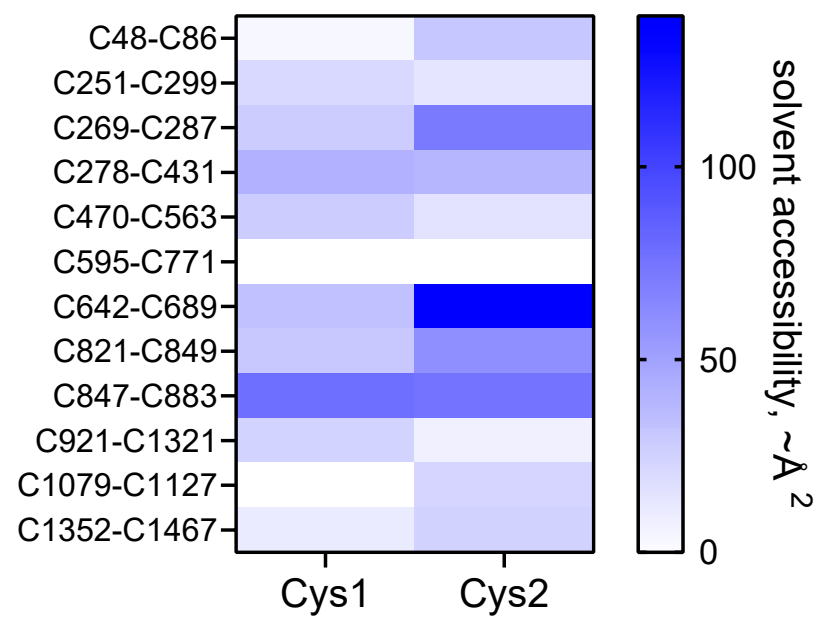

Figure 4. Heat map of the solvent accessibility of the $24 \alpha 2$-macroglobulin disulfide bond cysteines in the homo-tetramer. The scale is the solvent accessibility in $\sim \AA^{2}$ derived from DSSP [12] using the PDB identifier 4acq structure of $\alpha 2$-macroglobulin [5].

\section{Conclusions}

The conceptual and experimental challenges of dealing with many disulfide-bonded forms of a protein will be aided by theoretical estimates of the number of forms. This information will help investigators better define hypotheses and experimental strategies for tackling research questions. Future studies will refine the number and type of covalent states and explore how the different states contribute to protein function. The probabilities for bond formation approach outlined in this study is a starting point for estimating the number of disulfide-bonded forms of a protein.

Funding: This research was funded by the National Health and Medical Research Council of Australia, grant numbers 1110219,1143400 and 1143398.

Acknowledgments: I thank Aster Pijning and Mark Schreuder for helpful comments on the manuscript.

Conflicts of Interest: The author declares no conflict of interest. 


\section{References}

1. Wong, J.W.; Ho, S.Y.; Hogg, P.J. Disulfide bond acquisition through eukaryotic protein evolution. Mol. Biol. Evol. 2011, 28, 327-334. [CrossRef] [PubMed]

2. Butera, D.; Hogg, P.J. Fibrinogen function achieved through multiple covalent states. Nat. Commun. 2020, 11, 5468. [CrossRef] [PubMed]

3. Barrett, A.J.; Starkey, P.M. The interaction of alpha 2-macroglobulin with proteinases. Characteristics and specificity of the reaction, and a hypothesis concerning its molecular mechanism. Biochem. J. 1973, 133, 709-724. [CrossRef] [PubMed]

4. Garcia-Ferrer, I.; Marrero, A.; Gomis-Ruth, F.X.; Goulas, T. Alpha2-Macroglobulins: Structure and Function. Subcell. Biochem. 2017, 83, 149-183. [PubMed]

5. Marrero, A.; Duquerroy, S.; Trapani, S.; Goulas, T.; Guevara, T.; Andersen, G.R.; Navaza, J.; Sottrup-Jensen, L.; Gomis-Ruth, F.X. The crystal structure of human alpha2-macroglobulin reveals a unique molecular cage. Angew. Chem. Int. Ed. 2012, 51, 3340-3344. [CrossRef] [PubMed]

6. Schmidt, B.; Ho, L.; Hogg, P.J. Allosteric disulfide bonds. Biochemistry 2006, 45, 7429-7433. [CrossRef] [PubMed]

7. Chiu, J.; Hogg, P.J. Allosteric disulfides: Sophisticated molecular structures enabling flexible protein regulation. J. Biol. Chem. 2019, 294, 2949-2960. [CrossRef] [PubMed]

8. Zhou, B.; Baldus, I.B.; Li, W.; Edwards, S.A.; Grater, F. Identification of allosteric disulfides from prestress analysis. Biophys. J. 2014, 107, 672-681. [CrossRef] [PubMed]

9. Wong, J.W.; Hogg, P.J. Analysis of disulfide bonds in protein structures. J. Thromb. Haemost. 2010, 8, 2345. [CrossRef] [PubMed]

10. Hogg, P.J. Disulfide bonds as switches for protein function. Trends Biochem. Sci. 2003, 28, 210-214. [CrossRef]

11. Flaumenhaft, R.; Furie, B. Vascular thiol isomerases. Blood 2016, 128, 893-901. [CrossRef]

12. Kabsch, W.; Sander, C. Dictionary of protein secondary structure: Pattern recognition of hydrogen-bonded and geometrical features. Biopolymers 1983, 22, 2577-2637. [CrossRef]

Publisher's Note: MDPI stays neutral with regard to jurisdictional claims in published maps and institutional affiliations.

(C) 2020 by the author. Licensee MDPI, Basel, Switzerland. This article is an open access article distributed under the terms and conditions of the Creative Commons Attribution (CC BY) license (http://creativecommons.org/licenses/by/4.0/). 